\title{
Frequency and Pattern of Bone Marrow Infiltration in Classical Hodgkin's Lymphoma: Experience from Southern Pakistan
}

\author{
Sadia Sultan ${ }^{1 *}$, Syed Mohammed Irfan ${ }^{1}$, Saira Parveen ${ }^{1}$, Syeda Amna Haider ${ }^{2}$, \\ Mahira Masood ${ }^{2}$
}

\begin{abstract}
Background: Hodgkin's lymphoma (formerly, Hodgkins disease) is a potentially curable malignancy with distinctive biological behavior and specific clinical characteristics. Limited information is available from developing countries for patients with classical Hodgkin's lymphoma (cHL). Therefore we reviewed the demographical and clinico-hematological profiles along with bone marrow infiltration patterns in adult patients presenting at Liaquat National Hospital and Medical College. Materials and Methods: In this cross sectional study, 62 adult ( $\geq 15$ years) patients with $\mathrm{cHL}$ were enrolled from January 2010 to December 2014. Results: The mean age was $29.7 \pm 13.8$ years with a median of 30 years. The male to female ratio was $2: 1$. B symptoms were present in $72.5 \%$ of patients and lymph node enlargement in $85.4 \%$. The frequency of bone marrow infiltration in our cHL patients was found to be $27.4 \%$, the pattern being predominantly focal followed by diffuse. The mean hemoglobin was $9.4 \pm 1.9 \mathrm{~g} / \mathrm{dl}$ with a mean MCV of $78.1 \pm 7.9 \mathrm{fl}$, a mean total leukocyte count of $10.9 \pm 20.6 \times 10^{9} / \mathrm{l}$ and a mean platelet count of $241.6 \pm 150.1 \times 10^{9} /$. Conclusions: Our analysis shows that clinico-pathological features of cHL in Pakistan are comparable to published data. Peripheral lymphodenopathy associated with B symptoms is the commonest presentation. Bone marrow involvement is more common in our setup as patients usually presented at an advanced stage of disease.
\end{abstract}

Keywords: Hodgkin's lymphoma - clinico-pathology - bone marrow infiltration -Pakistan

Asian Pac J Cancer Prev, 17 (4), 1857-1859

\section{Introduction}

Classical Hodgkin lymphoma (cHL) encompasses a group of lymphoid neoplasms with a multifaceted etiology and epidemiology (Roshandel et al., 2011). As per WHO, cHL accounts for about $1 \%$ of all cancers and $230 \%$ of the lymphoid malignancies worldwide (Swerdlow et al., 2008). Literature review demonstrates a remarkable diversity of the disease incidence according to age, gender, ethnic background, geographical location and socioeconomical status (Jarrett et al., 1996; Cartwright et al., 2004). The highest incidence was reported among Caucasians, followed by African Americans and Hispanics (Cartwright et al., 2004).

Hodgkin lymphoma is an uncommon malignancy with an overall incidence of about 2.4-2.8/100,000 individuals yearly with the peak cases reported between 15-29 years of age (Sive and Linch., 2011; Smith et al., 2015; SaderGhorra et al., 2014). Recent advancement in diagnosis and treatment of Hodgkin's lymphoma has changed the perspective of disease.

Though no clear cut etiological agent has been identified, but the association between Hodgkin's lymphoma and viral infections has been discussed, especially regarding Epstein Barr Virus infection and human immunodeficiency virus (Tang et al., 2013; Li et al., 2012).

The clinical biology of cHL among patients is highly heterogeneous depending upon disease stage at presentation. These symptoms often include fever, drenching sweating, weight loss, fatigue or peripheral lymphodenopathy. Visceromegaly may occur in these patients, but it is typically mild and mostly asymptomatic.

Hodgkin lymphoma (cHL) is characterized morphologically by the presence of distinctive pathognomonic cells called Reed Sternberg (RS) cells in the background of reactive lymphocytes, histiocytes (macrophages) and granulocytes. Bone marrow involvement by Hodgkin lymphoma is rather uncommon in western studies (Howell et al., 2002; Levis et al., 2004). The involvement of the marrow in a patient with classical Hodgkin lymphoma represents advanced stage IV disease.

To date very little information is available from our country on this disorder. Research on epidemiological 


\section{Sadia Sultan et al}

features of $\mathrm{cHL}$ is poorly reported from countries with limited resources. This report describes the demographical data, clinical features and laboratory findings in cHL patients. Secondly we also determined the frequency and pattern of bone marrow infiltration by $\mathrm{cHL}$ at the time of diagnosis.

\section{Materials and Methods}

This retrospective cross sectional study was conducted at hematology department of Liaquat National Hospital, extended over a period of 5 years from January 2010 to December 2014.

The cases were diagnosed by morphology on $\mathrm{H} \& \mathrm{E}$ sections and IHC profile according to WHO classification of lymphoid neoplasms. Immunohistochemical (IHC) stains using antibodies against CD 20, CD 3, CD 10, CD 5, CD 23, BCL2, cyclin D1, CD 79a, CD 30 and CD 15 were done in each case. Patients diagnosed as classical HL (CD $15 \& 30$ positivity) who were $\geq 15$ years of age were included in the study. Patients with other lymphoid neoplasm were excluded. Patients with another associated malignancy or having relapsed/ refractory HL were also excluded. All post-chemotherapy and radiotherapy cases were not included in the study.

Based on this, a total of 62 subjects with newly diagnosed untreated classical HL were included in this analysis.

Complete blood counts were determined by Automated Cell Dyne counter. Bone marrow aspirate and trephine samples were taken from posterior superior iliac spine by the standard technique through Jamshidi needle after informed written consent. Trephine sections were stained with $\mathrm{H} \& \mathrm{E}$, which was reviewed by expert hematologist.

An ethical approval to conduct this analysis was granted by the institutional ethical review committee.

\section{Statistics analysis}

Data was assembled and analyzed using the Statistical Package for the Social Sciences version 22.0 (SPSS Inc, Chicago, IL, USA). The results were expressed as mean (SD) for quantitative variables and are presented as frequency \& percentages for qualitative variables.

\section{Results}

\section{Baseline characteristics}

Out of 62 patients, 42 were males $(67.7 \%)$ and 20 were females $(32.2 \%)$ with male to female ratio of $2: 1$. Age ranged between 15 and 76 years with the mean age was $29.7 \pm 13.8$ years and the median age of 30 years. Majority of patients $(74.1 \%)$ were under 30 years of age (table-1).

\section{Clinical findings}

B symptoms were present in $45(72.5 \%)$ patients, out of which fever was commonest, seen in 40 (64.5\%); drenching night sweats in $36(55 \%)$ patients, while 23 $(37 \%)$ patients had history of weight loss. Lymph node enlargement was present in $53(85.4 \%)$ cases. The most common lymph node palpable was cervical and axillary nodes. The splenomegaly and hepatomegaly were noted
Table 1. Age Stratification in Adult Patients with Hodgkin's lymphoma

\begin{tabular}{ccccc}
\hline \multirow{2}{*}{ Age groups } & Male & Female & Total & Percentage \\
\cline { 2 - 5 } & $\mathrm{n}=42$ & $\mathrm{n}=20$ & $\mathrm{n}=62$ & $\%$ \\
\hline $15-30$ & 31 & 15 & 46 & 74.1 \\
$31-50$ & 5 & 3 & 8 & 12.9 \\
$51-65$ & 4 & 2 & 6 & 9.6 \\
$65-76$ & 2 & 0 & 2 & 3.2 \\
\hline
\end{tabular}

in $16(25.8 \%)$ and $11(17.7 \%)$ patients respectively.

\section{Laboratory features}

The frequency of bone marrow infiltration in our cHL patients was found to be $27.4 \%(n=17)$. Pattern of infiltrate was focal in $9(14.5 \%)$ patients followed by diffuse in 7 (11.2\%) patients. The least encountered type was nodular in $1(1.7 \%)$ of patient.

The mean hemoglobin was $9.4 \pm 1.9 \mathrm{~g} / \mathrm{dl}(6.8-15.8 \mathrm{~g} /$ dl) with the mean MCV of $78.1 \pm 7.9 \mathrm{fl}$. The mean total leukocyte count of $10.9 \pm 20.6 \times 10^{9} / 1\left(1.5-22.9 \times 10^{9} / 1\right)$; mean absolute neutrophils count of $3.8 \pm 3.0 \times 10^{9} / 1$ and the mean platelets count were $241.6 \pm 150.1 \times 10^{9} / 1$ (16-651 x10 $\left./ 1\right)$. Anemia $(\mathrm{Hb}<10 \mathrm{gm} / \mathrm{dl})$ was noted in $15(24.1 \%)$ patients. Thrombocytopenia (platelets count $<100 \times 10^{9} / 1$ ) was detected in $5(8.0 \%)$ patients.

\section{Discussion}

Classical Hodgkin's lymphoma is an uncommon B-cell lymphoid neoplasm which affect around 9000 new patients per annum (Ansell., 2015). The prognosis of patients with classical Hodgkin lymphoma has been tremendously improved during the last 4 decades. cHL is an uncommon entity and very limited studies are available from Pakistan. Herein we have described a series of 62 adults cHL patients followed at our institution during the five year period.

The principal cHL epidemiological features so far described show age predilection in more studies, with the median age around $\sim 30$ years. In Pakistani population disease is also identified in younger age group, as majority (3/4) of our patients were under 30 years. This is consistent with previous reports published from Pakistan on cHL by Siddiqui and Fatima et al (Siddiqui et al., 2006; Fatima et al., 2011). This is also similar to earlier studies from India and Egypt where average age were 30 and 31.4 years respectively (Ganesan et al., 2011; Gad Allah et al., 2014). However, we did not observe a second peak of age as have been noted in earlier studies from developed countries (Hasenclever et al., 1998).

It is consistently noted that $\mathrm{cHL}$ is frequently seen in male patients. Higher male to female ratio has also been established in earlier studies from Pakistan and other countries include Korea and Brazil (Cheong et al., 2006; Vassallo et al., 2005).

In general, Hodgkin lymphoma is characterized by the customary spread of disease from one lymph node group to another and by the development of B-systemic symptoms usually associated with advanced disease. 
In the present study majority patients presented with intermittent fever, followed by night sweats and significant weight loss. It was noted that the prevalence of B-symptoms in our patients from southern Pakistan was comparable with that reported in the previous local study (fever; $59.6 \%$, weight loss; $33.6 \%$ ) from Pakistan (Siddiqui et al., 2006). Majority (85.4\%) of our patients presented with lymph node enlargement. This is consistent with previous reports published from India and turkey on classical HL (Ganesan et al., 2011; Kılıçkap et al., 2013).

Staging of the cHL is foremost important for the therapeutic decisions in the management of these patients. Bone trephine biopsy is one of the important investigation, which is being carried out for staging workup along with other investigations like CBC, Computed tomography and PET scanning. Bone marrow infiltration in $\mathrm{cHL}$ has been observed from various racial backgrounds ranging from $2 \%$ to as high as $38 \%$ (Lone et al., 2011).

Previous studies reported from Pakistan revealed relatively higher prevalence of lymphomatous infiltration compared with western studies. Our findings are comparable with those of Butt $(27.5 \%)$, Lone (38\%) and Hamid (30\%) reported from different areas from Pakistan (Butt et al., 2002; Lone et al., 2011; Hamid et al., 2010).

In contrast, studies from Italy and United Kingdom revealed fewer prevalence of infiltration accountable in $10 \%$ and 2.2\% respectively (Franco et al., 2004; Howell et al., 2002). Distinctly in economically developed countries where majority of patients present with early stage (stage I and II), 27.4\% of patients at our center presented with advanced stage (Shankar et al., 1997). A higher percentage of bone marrow infiltration in Pakistan may be explained on the basis that $\sim 1 / 3$ patients present at late stages of disease in contrast to developed countries. The delay in seeking medical treatment is attributed to financial constraints and lack of diagnostic and referral facilities.

Lastly limitations of our study need to be mentioned, related to its retrospective nature and relatively small sample study. A larger sample size study would be a better indicator of clinical and laboratory attributes in our patients population. The results are from a single tertiary care centre and consequently, there may be a referral bias.

In conclusion, the present study reflects the invasive potential of classical Hodgkin's lymphomas in our population. Important observations in the present study include young age at diagnosis, higher male to female ratio, higher incidence of $\mathrm{B}$ symptoms and higher proportion of patients with advanced cHL having marrow infiltration at disease manifestation.

\section{References}

Ansell SM (2015). Hodgkin lymphoma: diagnosis and treatment. Mayo Clin Proc, 90, 1574-83.

Butt F, Akhtar R, Rahmani T, et al (2002). Bone marrow involvement in lymphoma: Incidence and co-relation with age and sex. Biomedica, 18, 53-7.

Cartwright RA, Watkins G (2004). Epidemiology of Hodgkin's disease: a review. Hematol Oncol, 22, 11-26.

Cheong JW, Park SY, Roh JK, et al (2006). Treatment of Hodgkin's disease: a twenty-year follow-up of patients at a center in Korea. Yonsei Med J, 47, 455-65.
Fatima S, Ahmed R, Ahmed A (2011). Hodgkin lymphoma in Pakistan: an analysis of subtypes and their correlation with Epstein Barr virus. Asian Pac J Cancer Prev, 12, 1385-8.

Franco V, Tripodo C, Rizzo A, et al (2004). Bone marrow biopsy in Hodgkin's lymphoma. Eur J Haematol, 73, 149-55.

Ganesan P, Kumar L, Raina V (2011). Hodgkin's lymphomalong-term outcome: an experience from a tertiary care cancer center in North India. Ann Hematol, 90, 1153-60.

Gad Allah H, El-Azzazi MO, Elafifi AM, et al (2014). Treatment outcome in Egyptian lymphoma patients, 2-year results, single-center experience. Egypt J Haematol, 39, 209-16.

Hasenclever D, Diehl V (1998). A prognostic score for advanced Hodgkin's disease. international prognostic factors project on advanced hodgkin's disease. $N$ Engl J Med, 339, 1506-14.

Hamid A, Hamid Am, Jabbar N, et al (2010). Significance of bone marrow biopsy in staging of Hodgkin's lymphoma. Ann King Edward Med University, 16, 12-16.

Howell SJ, Grey M, Chang J, et al (2002). The value of bone marrow examination in the staging of Hodgkin's lymphoma: a review of 955 cases seen in a regional cancer centre. $\mathrm{Br} \mathrm{J}$ Haematol, 119, 408-11.

Jarrett AF, Armstrong AA, Alexander E (1996). Epidemiology of EBV and Hodgkin's lymphoma. Ann Oncol, 7, 45-10.

Kılıçkap S, Barışta I, Ulger S, et al (2013). Clinical features and prognostic factors of hodgkin's lymphoma: a single center experience. Balkan Med J, 30, 178-85.

Li X, Yang SE, Guo YQ, et al (2012). Clinical significance of quantitative analysis of plasma epstein- barr virus DNA in patients of Xinjiang Uygur nationality with Hodgkin's Lymphoma. Asian Pac J Cancer Prev, 13, 6379-84.

Levis A, Pietrasanta D, Godio L, et al (2004). A large-scale study of bone marrow involvement in patients with Hodgkin's lymphoma. Clin Lymphoma, 5, 50-5.

Lone A, Naeem S (2011). Frequency and pattern of bone marrow infiltration in Hodgkin's Lymphoma. Biomedica, 27, 132-5.

Roshandel G, Semnani S, Aarabi M, et al (2011). Lymphomas in Golestan province of Iran: results of a population-based cancer registry. Asian Pac J Cancer Prev, 12, 219-22.

Sader-Ghorra C, Rassy M, Naderi S, et al (2014).Type distribution of lymphomas in Lebanon: five-year single institution experience. Asian Pac J Cancer Prev, 15, 5825-8.

Shankar AG, Ashley S, Radford M, et al (1997). Does histology influence outcome in childhood Hodgkin's disease? Results from the United Kingdom Children's Cancer Study Group. J Clin Oncol, 15, 2622-30.

Siddiqui N, Ayub B, Badar F, et al (2006). Hodgkin's lymphoma in Pakistan: a clinico-epidemiological study of 658 cases at a cancer center in Lahore. Asian Pac J Cancer Prev, 7, 651-5.

Smith A, Crouch S, Lax S,et al (2015). Lymphoma incidence, survival and prevalence 2004-2014: sub-type analyses from the UK's haematological malignancy research network. $\mathrm{Br}$ J Cancer, 112, 1575-84.

Sive J, Linch D (2011). Hodgkin lymphoma. In:Hoffbrand AV, Catovsky D, Tuddenham EG, Green AR. Post graduate hematology, $6^{\text {th }}$ edition, 639 .

Swerdlow SH, Campo E, Harris NL, et al (2008). WHO Classification of Tumors: Pathology and Genetics of Tumors of Haematopoietic and Lymphoid Tissues. Lyon: IARC Press, 321-34.

Tang Y, Sun LG, Liu CS, et al (2013). Clinical analysis of stages of HBV infection in 100 cases of lymphoma. Asian Pac J Cancer Prev, 14, 959-62.

Vassallo J, Paes RP, Soares FA, et al (2005). Histological classification of 1,025 cases of Hodgkin's lymphoma from the State of São Paulo, Brazil. Sao Paulo Med J, 123, 134-6. 